\title{
Rates of Bone Loss in the Appendicular and Axial Skeletons of Women Evidence of Substantial Vertebral Bone Loss Before Menopause
}

\author{
B. Lawrence Riggs, Heinz W. Wahner, L. Joseph Melton III, Linda S. Richelson, Howard L. Judd, and Kenneth P. Offord \\ Endocrine Research Unit, Division of Endocrinology, Metabolism, and Internal Medicine; Section of Diagnostic Nuclear Medicine, and \\ Department of Medical Statistics and Epidemiology, Mayo Clinic and Mayo Foundation, Rochester, Minnesota 55905; and University of \\ California at Los Angeles, School of Medicine, Center for the Health Services, Los Angeles, California 90024
}

\begin{abstract}
We made longitudinal measurements of bone mineral density (BMD) in 139 normal women (ages 20-88 yr) at midradius (99\% cortical bone) and lumbar spine ( $\sim 70 \%$ trabecular bone) by single- and dual-photon absorptiometry. BMD was measured 2-6 (median, 3) times over an interval of 0.8-3.4 yr (median, 2.1 yr). For midradius, BMD did not change $(+0.48 \% / y r, N S)$ before menopause but decreased $(-1.01 \% / y r, P<0.001)$ after menopause. For lumbar spine, there was significant bone loss both before $(-1.32 \% / y r, P<0.001)$ and after $(-0.97 \% / y r, P=0.006)$ menopause; these rates did not differ significantly from each other. Our data show that before menopause little, if any, bone is lost from the appendicular skeleton but substantial amounts are lost from the axial skeleton. Thus, factors in addition to estrogen deficiency must contribute to pathogenesis of involutional osteoporosis in women because about half of overall vertebral bone loss occurs premenopausally.
\end{abstract}

\section{Introduction}

Osteoporosis is a common problem among the elderly and is associated with a high incidence of skeletal fractures, especially of the vertebrae and hip (1). Women are more susceptible than men (1). Bone densitometric studies suggest that osteoporosis is the result of a long period of slow bone loss, and the patterns may differ between cortical bone and trabecular bone $(2,3)$. Precise methods for measuring bone loss in the predominantly cortical bone of the appendicular skeleton have been available for almost $20 \mathrm{yr}$, and a number of cross-sectional and longitudinal studies have defined the pattern of cortical bone loss and the factors that influence it $(2,4)$. By contrast, reliable methods for measuring bone loss from the predominantly trabecular bone of the axial skeleton have become available only recently (4). Thus, the longitudinal pattern of axial skeletal loss is less well understood and is more controversial.

To define the pattern of age-related bone loss in women, we assessed rates of loss from the appendicular and axial skeletons by making repeated measurements over time in a large group of normal women whose ages ranged from 20 to $88 \mathrm{yr}$. To define the relationship with estrogen deficiency, we compared rates of

Address reprint requests and correspondence to Dr. Riggs, Endocrine Research Unit, Division of Endocrinology, Metabolism, and Internal Medicine, Mayo Clinic, 200 First Street, SW, Rochester, MN 55905. 1986.

Received for publication 9 August 1985 and in revised form 28 January

J. Clin. Invest.

(c) The American Society for Clinical Investigation, Inc.

$0021-9738 / 86 / 05 / 1487 / 05 \quad \$ 1.00$

Volume 77, May 1986, 1487-1491 bone loss before and after menopause and assessed the correlation with serum concentrations of sex steroids.

\section{Methods}

Experimental subjects and protocol. We made bone mineral density (BMD) ${ }^{1}$ measurements at the midradius and lumbar spine in 139 normal Caucasian women who were residents of Rochester, MN. Of these, 92 women were from a stratified random sample of the community population (as part of an ongoing epidemiologic study) and the remainder were local volunteers. The median age was 52 yr (range, 20-88 yr). Normality was assessed by interview, by review of each patient's medical records, and by laboratory testing. All subjects were ambulatory. None had vertebral fractures on roentgenograms of the spinal column or a history of hip fracture. None were taking corticosteroids, anticonvulsant medication, thiazide diuretics, vitamin $\mathrm{D}$ in pharmacologic doses, or calcium supplements at dosages $>500 \mathrm{mg} / \mathrm{d}$. None of the postmenopausal women were taking estrogen replacement therapy. None had any evident medical disease known to affect calcium metabolism.

Menopause was defined as absence of menses for at least 6 mo and serum level of estradiol $<50 \mathrm{pg} / \mathrm{ml}$. There were 29 women who had had previous hysterectomy; for the 7 of these who had had bilateral oophorectomy, the date of menopause was taken as the date of this operation. For the remaining 22 women who had had only a hysterectomy or hysterectomy plus unilateral oophorectomy, postmenopausal status was defined by the serum estradiol level $(<50 \mathrm{pg} / \mathrm{ml})$. Values of serum estradiol below this level were chosen arbitrarily as indicating decreased residual ovarian function, but the date of onset of menopause in such women was indeterminate. By these definitions, 59 women were premenopausal and 80 were postmenopausal at baseline.

Fasting-state blood samples were drawn for determination of serum sex steroid levels. For the premenopausal women, the blood sample was obtained between days 7 and 10 of the menstrual cycle. Bone densitometry was repeated on one or more occasions, and the interval between the first and last measurement ranged from 0.8 to $3.4 \mathrm{yr}$ (median, $2.1 \mathrm{yr}$ ). The number of measurements per subject ranged from 2 to 6 (median, 3).

All subjects gave their informed consent. The experimental protocol was reviewed and approved by the Mayo Institutional Review Board.

Laboratory methods. BMD of the midradius was measured by singlephoton absorptiometry as described elsewhere (5) except that the scanner was modified to permit rectilinear scanning. The recorded value was the mean of five scan passes made at intervals of $0.1 \mathrm{~cm}$. Reproducibility $(\mathrm{SD} /$ mean of repeat measurements $\times 100)$ was $1 \%$. BMD of the lumbar spine was measured by dual photon absorptiometry (6); reproducibility was $2.2 \%$. The approximate composition of bone is $99 \%$ cortical for the midradius and $>70 \%$ trabecular for the lumbar spine (3).

Serum estrone, estradiol, testosterone, androstenedione, and dehydroepiandrosterone were measured by previously described radioimmunoassay techniques (7). Serum sex hormone-binding globulin was assayed by the ammonium sulfate precipitation technique as described by Rosner (8). A partial correction was made for differences in total serum concentrations of sex steroids due to differences in sex hormone

1. Abbreviations used in this paper: BMD, bone mineral density. 
binding by dividing the former by the concentration of the binding globulin.

Statistical methods. To assess rates of bone loss at either scanning site, a straight-line regression for BMD on age was fitted separately for each subject by the method of least squares. The dependent variable $(y)$ was BMD and the independent variable $(x)$ was time, in years, from baseline measurement. The slope at midradius was expressed in units of $\mathrm{g} / \mathrm{cm}$ per yr and at lumbar spine, in units of $\mathrm{g} / \mathrm{cm}^{2}$ per yr. Each slope was divided by its $y$-intercept at baseline and multiplied by 100 to obtain an estimate of percentage change per year. The changes in rate of bone loss with age were assessed by successively fitting linear, parabolic, cubic, and quartic polynomial equations on age. The significances of the regression coefficients were then examined. Variables affecting rates of bone loss were evaluated by multiple linear regression analysis. The relationship of several independent variables to the dependent variable of rate of bone loss was assessed by standard statistical methods for multiple linear regression. Two- and one-sample $t$ tests also were performed. All $P$ values were two-tailed. The Statistical Analysis System (9) was used to carry out all statistical computations.

\section{Results}

Bone densitometry. The results obtained by expressing the slopes in absolute terms (in $\mathrm{g} / \mathrm{cm}$ per yr or $\mathrm{g} / \mathrm{cm}^{2}$ per $\mathrm{yr}$ ) and in relative terms (in \%/yr) were found to be highly correlated ( $r=0.99$ for midradius and $r=0.985$ for lumbar spine). Thus, we elected to express results as \%/yr for the sake of simplicity.

For the midradius, the rate of change in BMD with age was best described by a linear function (Fig. 1). Considering all subjects, for the lumbar spine, the rate of bone loss did not vary with age; overall mean rate of change was $-1.12 \% / \mathrm{yr}(P<0.001)$.

Inspection of densitometric values for midradius and lumbar spine showed that, when the subjects were grouped according to years of study, there was a progressive decrease in variability between subjects of these longitudinal rates of change with increases in study interval (from $\sim 1 \mathrm{yr}$ [range, $0.8-1.3 \mathrm{yr}$ ] to $\sim 2$ yr [range, 1.4-2.7 yr] to $\sim 3$ yr [range, 2.8-3.4 yr]). Thus, the variation in rate of change about the lumbar spine regression line with age $\left(S_{y \cdot x}\right)$ was $4.1,2.6$, and $1.1 \% / y r$, respectively, for the measurement intervals of $\sim 1,2$, and $3 \mathrm{yr}$. Accordingly, the

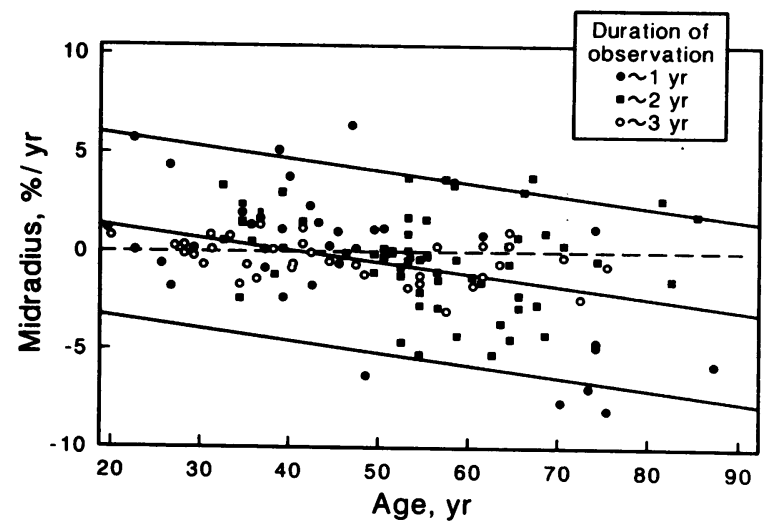

Figure 1. Rate of change in BMD of midradius (\%/yr) in 139 normal women plotted against age at time of first BMD measurement. Subjects are identified by time on study. Age regression was best fitted with linear equation $\left(y=2.806-0.064 x, \mathrm{~S}_{\mathrm{y} \cdot \mathrm{x}}=2.30 ; r^{2}=0.15\right)$. Center line denotes age regression; upper and lower lines denote $95 \%$ confidence limits. Broken line indicates zero rate of change in BMD.
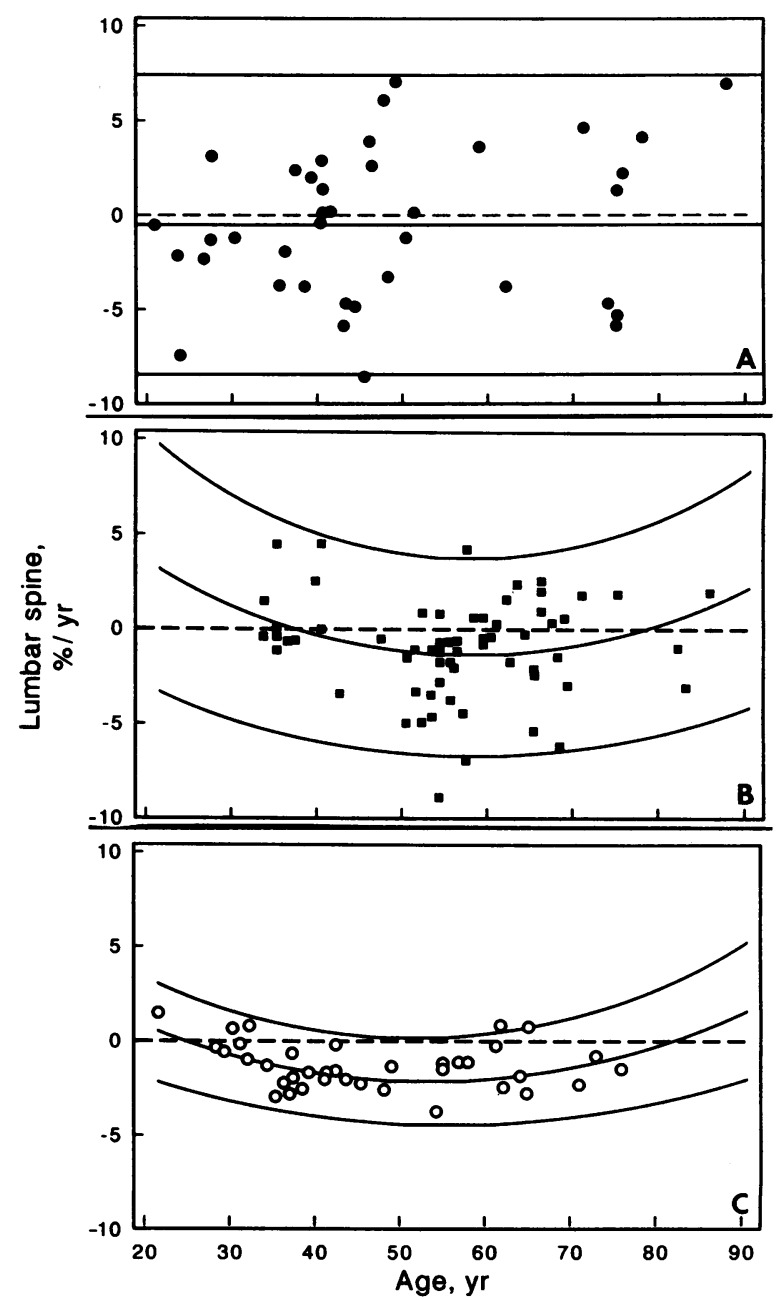

Figure 2. Rate of change in BMD of lumbar spine in 139 normal women plotted against age at time of first BMD measurement. Subjects are identified by time on study: $(A) \sim 1 \mathrm{yr}\left(y=-0.71 ; \mathrm{SD}_{\mathrm{y}}\right.$ $=4.11) ;(B) \sim 2 \operatorname{yr}\left(y=10.110-0.403 x+0.003 x^{2} ; \mathrm{S}_{\mathrm{y} \cdot \mathrm{x}}=2.62 ; r^{2}\right.$ $=0.08) ;(C) \sim 3 \mathrm{yr}\left(y=5.039-0.280 x+0.003 x^{2} ; \mathrm{S}_{\mathrm{y} \cdot \mathrm{x}}=1.13 ; r^{2}\right.$ $=0.23$ ). When all data were analyzed, mean bone loss occurred across life. When only subjects in the groups having mean scan intervals of $\sim 2$ yr or $\sim 3$ yr were analyzed, however, age regression was best fitted with the parabolic equation. Center line denotes age regression and upper and lower lines denote $95 \%$ confidence limits for this equation. Broken line shows zero rate of change in BMD.

women were categorized by length of time on the study and their data were reanalyzed. For the lumbar spine, the parabolic fit was significantly better than the linear fit for subjects in the 2 -yr $(P<0.01)$ and 3-yr $(P<0.001)$ intervals but not in the 1 yr interval; rates of bone loss were maximal during middle life (Fig. 2).

The effect of the menopause on bone loss was assessed by comparing rates of change in premenopausal and postmenopausal women. For midradius, there was no significant association between rate of change and age in the premenopausal women ( $r=-0.19$, NS), whereas a significant linear trend was observed for the postmenopausal women $(r=-0.24, P<0.05)$. When the mean rates of change for the midradius were compared, there was greater $(P<0.001)$ bone loss in the postmenopausal than in the premenopausal women. Using all women, 
Table I. Rates of Change in BMD Grouped by Interval of Study and Menopausal Status

\begin{tabular}{|c|c|c|c|c|c|c|c|c|}
\hline & \multicolumn{8}{|c|}{ Rate of change in BMD } \\
\hline & \multicolumn{2}{|l|}{ Overall } & \multicolumn{2}{|c|}{$\sim$ 1-yr Interval } & \multicolumn{2}{|c|}{$\sim 2$-yr Interval } & \multicolumn{2}{|c|}{ ３-yr Interval } \\
\hline & $\begin{array}{l}\text { Pre } \\
(n=59)\end{array}$ & $\begin{array}{l}\text { Post } \\
(n=80)\end{array}$ & $\begin{array}{l}\text { Pre } \\
(n=22)\end{array}$ & $\begin{array}{l}\text { Post } \\
(n=16)\end{array}$ & $\begin{array}{l}\text { Pre } \\
(n=13)\end{array}$ & $\begin{array}{l}\text { Post } \\
(n=50)\end{array}$ & $\begin{array}{l}\text { Pre } \\
(n=24)\end{array}$ & $\begin{array}{l}\text { Post } \\
(n=14)\end{array}$ \\
\hline & percent/yr & percent/yr & percent/yr & percent/yr & percent $/ y r$ & percent/yr & percent/yr & percent/yr \\
\hline \multicolumn{9}{|l|}{ Midradius } \\
\hline Mean & +0.48 & $-1.15 \S$ & +0.93 & $-2.15^{*}$ & +0.85 & $-0.87 \ddagger$ & -0.14 & $-1.03^{*}$ \\
\hline SD & 1.69 & 2.75 & 2.18 & 4.50 & 1.71 & 2.29 & 0.79 & 1.15 \\
\hline Range & $\begin{array}{r}-2.49 \text { to } \\
+5.75\end{array}$ & $\begin{array}{r}-8.49 \text { to } \\
+6.07\end{array}$ & $\begin{array}{r}-2.49 \text { to } \\
+5.75\end{array}$ & $\begin{array}{r}-8.49 \text { to } \\
+6.07\end{array}$ & $\begin{array}{c}-2.49 \text { to } \\
+3.37\end{array}$ & $\begin{array}{r}-5.31 \text { to } \\
+3.43\end{array}$ & $\begin{array}{r}-1.71 \text { to } \\
+1.30\end{array}$ & $\begin{array}{r}-3.10 \text { to } \\
+0.85\end{array}$ \\
\hline \multicolumn{9}{|c|}{ Lumbar spine } \\
\hline Mean & -1.32 & -0.97 & -1.88 & $+0.89^{*}$ & +0.17 & -1.35 & -1.61 & -1.76 \\
\hline SD & 2.61 & 3.08 & 3.44 & 4.52 & 2.50 & 2.67 & 1.22 & 1.33 \\
\hline Range & $\begin{array}{c}-9.07 \text { to } \\
+4.59\end{array}$ & $\begin{array}{c}-9.04 \text { to } \\
+7.08\end{array}$ & $\begin{array}{r}-9.07 \text { to } \\
+4.59\end{array}$ & $\begin{array}{c}-6.03 \text { to } \\
+7.08\end{array}$ & $\begin{array}{c}-3.50 \text { to } \\
+4.59\end{array}$ & $\begin{array}{r}-9.04 \text { to } \\
+4.14\end{array}$ & $\begin{array}{r}-3.36 \text { to } \\
+1.24\end{array}$ & $\begin{array}{r}-4.22 \text { to } \\
+0.44\end{array}$ \\
\hline
\end{tabular}

Pre, premenopausal; post, postmenopausal. $* P<0.05 ; \ddagger P<0.01$; and $\S P<0.001$, for significance of difference between premenopausal and postmenopausal groups.

mean rates of change for lumbar spine BMD were significantly less than zero for both the premenopausal (mean, $-1.32 \% / \mathrm{yr}$, $P<0.001$ ) and the postmenopausal (mean, $-0.97 \% / \mathrm{yr}, P$ $=0.006$ ) women. Mean rates of change for lumbar spine before and after menopause were not significantly different. Table I gives rates of change in BMD for all subjects and subgroups by time on study and menopausal status.

Values of BMD $(\mathrm{g} / \mathrm{cm})$ at midradius and lumbar spine $(\mathrm{g} /$ $\left.\mathrm{cm}^{2}\right)$ were highly correlated $(r=0.72, P<0.001)$ at baseline. The rates of change $(\% / y r)$ at these two sites, however, differed markedly and there was no evidence of a correlation $(r=-0.04$, NS). These relationships are displayed in Figs. 3 and 4.

Sex steroids. Table II presents the correlation coefficients for levels of serum sex steroids with age. Serum estrone and estradiol decreased from high to low levels following menopause; however, there was no decrease before menopause. Fig. 5 gives individual values for serum estradiol in menstruating and nonmenstruating women. Multiple regression analysis was used to determine if levels of sex steroids could predict the rates of change

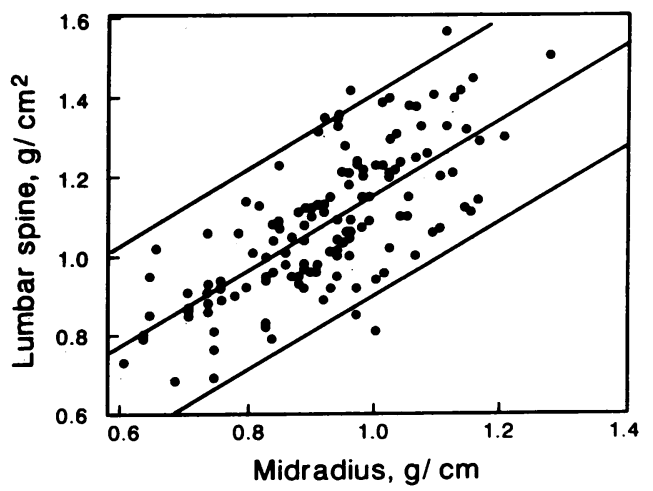

Figure 3. Relationship between baseline BMD values of lumbar spine (>70\% trabecular bone) and midradius (99\% cortical bone) in 139 normal women. Correlation is highly significant $(y=0.175+0.982 x$, $\left.\mathrm{S}_{\mathrm{y} \cdot \mathrm{x}}=0.13 ; r=0.72, P<0.001\right)$. in BMD after adjustment for the effect of age. Because of the possibility that the total serum levels of sex steroids were affected by changes in serum levels of sex-hormone binding globulin, we also entered into the equation the quotients of serum total concentration of estradiol, estrone, or testosterone divided by the concentration of serum sex hormone-binding globulin. With one exception, after adjusting for age, no variable or combination of serum sex steroids was found to predict the rate of bone loss at either midradius or lumbar spine when analyzed separately for premenopausal, for postmenopausal, or for all subjects. The one exception was a marginally lower $(P=0.03)$ ratio of levels of serum estrone/sex hormone binding globulin in those postmenopausal women with greater bone loss at midradius.

\section{Discussion}

As previously reported $(2,10)$, we found that levels of BMD in the appendicular and axial skeletons correlated well in normal women. The correlation with the radius, however, explains only

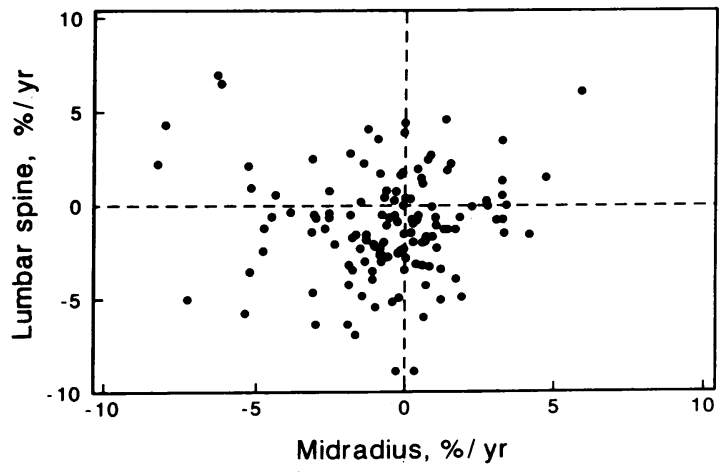

Figure 4. Relationship between longitudinal rates of change in BMD of midradius ( $99 \%$ cortical bone) and lumbar spine ( $>70 \%$ trabecular bone) in 139 normal women. There is no suggestion of a relationship $(r=-0.04, \mathrm{NS})$. 
Table II. Serum Sex Steroid Concentrations in 110 Normal Women* and Correlation with Age

\begin{tabular}{|c|c|c|c|c|c|c|}
\hline \multirow[b]{2}{*}{ Serum sex steroidł } & \multicolumn{2}{|c|}{ Overall $(n=110)$} & \multicolumn{2}{|c|}{ Premenopausal $(n=54)$} & \multicolumn{2}{|c|}{ Postmenopausal $(n=56)$} \\
\hline & Mean $( \pm \mathrm{SD})$ & $r \S$ & Mean $( \pm \mathrm{SD})$ & $r$ & Mean $( \pm \mathrm{SD})$ & $r$ \\
\hline$E 1-\log _{10}$ & $1.51 \pm 0.28$ & $-0.45^{* *}$ & $1.69 \pm 0.24$ & $0.41 \pi$ & $1.33 \pm 0.18$ & 0.11 \\
\hline $\mathrm{E} 1 / \mathrm{SHBG}-\log _{10}$ & $0.76 \pm 0.33$ & $-0.46^{* *}$ & $0.95 \pm 0.27$ & 0.09 & $0.58 \pm 0.27$ & 0.03 \\
\hline $\mathrm{E} 2-\log _{10}$ & $1.50 \pm 0.53$ & $-0.62^{* *}$ & $1.91 \pm 0.43$ & 0.26 & $1.09 \pm 0.23$ & -0.14 \\
\hline$E 2 / S H B G-\log _{10}$ & $0.75 \pm 0.55$ & $-0.65^{* *}$ & $1.17 \pm 0.40$ & 0.10 & $0.34 \pm 0.32$ & -0.14 \\
\hline TEST $-\log _{10}$ & $2.30 \pm 0.21$ & $-0.25 \pi$ & $2.34 \pm 0.18$ & $-0.31^{\prime \prime}$ & $2.26 \pm 0.23$ & -0.09 \\
\hline TEST/SHBG- $\log _{10}$ & $1.55 \pm 0.26$ & $-0.28 \pi$ & $1.59 \pm 0.25$ & $-0.50 \S$ & $1.51 \pm 0.26$ & -0.13 \\
\hline ANDR- $\log _{10}$ & $2.76 \pm 0.28$ & $-0.66^{* *}$ & $2.94 \pm 0.23$ & $-0.28^{\prime \prime}$ & $2.59 \pm 0.21$ & $-0.36 \pi$ \\
\hline DHEA- $\log _{10}$ & $0.44 \pm 0.33$ & $-0.48^{* *}$ & $0.58 \pm 0.31$ & -0.32 & $0.32 \pm 0.30$ & -0.29 \\
\hline SHBG & $5.98 \pm 2.40$ & 0.14 & $5.83 \pm 2.43$ & $0.34^{\prime \prime}$ & $6.12 \pm 2.38$ & 0.06 \\
\hline
\end{tabular}

* The 29 patients who had surgical menopause are not included in these data. $¥ E 1$, Estrone (pg/ml); SHBG, Sex hormone binding globulin $\times 10^{-8} \mathrm{M}$; E1/SHBG, ratio of E1 to SHBG; E2, Estradiol (pg/ml); E2/SHBG, ratio of E2 to SHBG; TEST, Testosterone (pg/ml); TEST/SHBG, ratio of TEST to SHBG; ANDR, Androstenedione (pg/ml); DHEA, dehydroepiandrosterone. $\$$ Linear correlation coefficient with age.

H.T,*** Refers to $P<0.05, P<0.01$, and $P<0.001$, respectively for the test of the null hypothesis of no correlation.

about half of the variance of the lumbar spine. By contrast, we found no significant relationship between rates of change of BMD in these two skeletal regions. This suggests the need for direct measurements to determine the rates of bone loss from the vertebrae.

In the most widely accepted model for age-related bone loss, there is no change in BMD in women from ages 25 to $40 \mathrm{yr}$; BMD then decreases minimally until the menopause, when large losses occur for at least a decade followed by a slowing of the rate of loss $(2,11,12)$. This model has been based almost entirely on BMD measurements in the predominantly cortical bone of the appendicular skeleton. Most workers have assumed that the pattern of bone loss from the predominantly trabecular bone of the axial skeleton follows a pattern that is qualitatively similar, although perhaps quantitatively greater.

For the midradius, our findings are consistent with this traditional model. We found no significant bone loss before age 50 $\mathrm{yr}$, or the menopause, but large losses thereafter. For the lumbar spine, we found that substantial premenopausal bone loss occurred. Mathematical fitting of the individual data points suggested bone loss throughout much of life with a trend toward midlife acceleration. Using all data, rates of bone loss were similar in the premenopausal and the postmenopausal women. Thus, our findings for the lumbar spine differ from those for the midradius and do not conform to the traditional model.

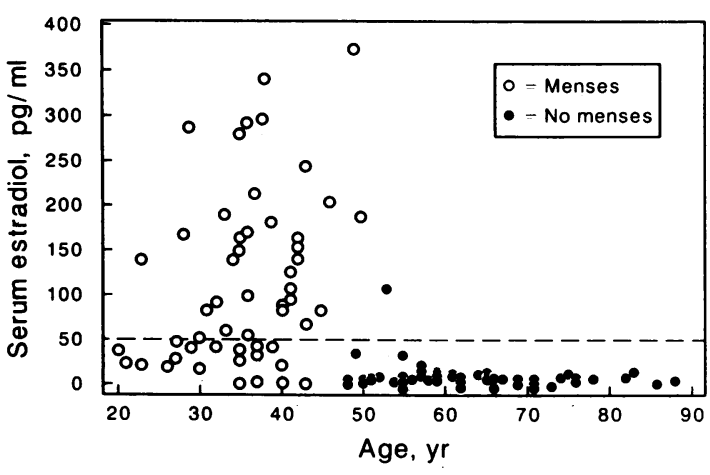

Figure 5. Baseline values for serum levels of estradiol plotted by age at first BMD measurement in 53 normal menstruating women (o) and 57 women who had had no menses for 6 mo or longer (๑). Subjects who have had hysterectomy are not shown.
These findings, however, are consistent with previous crosssectional densitometric studies in normal women that showed a linear $(6)$ or curvilinear $(13,14)$ decrease in BMD of the lumbar spine with aging and an onset well before midlife. Also, anatomic studies have shown a linear decrease in trabecular bone mass beginning in young adulthood, assessed either by iliac crest biopsy (15-17) or by postmortum examination of the vertebrae (18, 19). However, in a cross-sectional study, Aloia et al. (20) found that BMD of lumbar spine did not decrease in women until after age $50 \mathrm{yr}$. Ruegsegger et al. (21) found no significant loss of trabecular bone from the distal radius or tibia (as assessed by computed tomography) over a 24-mo period in 12 premenopausal women. Their finding suggests that patterns of trabecular bone loss in the appendicular and axial skeletons may differ.

A large body of data documents that menopause increases bone loss. After oophorectomy, accelerated bone loss that can be prevented or diminished by estrogen replacement has been demonstrated for both appendicular skeleton (22-24) and lumbar spine (25). Thus, bone loss over life from the axial skeleton may be the summation of estrogen-dependent and estrogen-independent mechanisms. Based on a densitometric comparison of a group of women (oophorectomized in young adulthood) with perimenopausal and postmenopausal control groups, we concluded previously that estrogen deficiency was the cause of most of age-related bone loss (26). From our new data it is clear that the experimental design of that study was inadequate because the perimenopausal control group had already undergone substantial bone loss before menopause. Also, it is possible that oophorectomy induces greater bone loss in young women in whom bone turnover is more active than in older women.

At present, we cannot explain why we did not detect accelerated postmenopausal bone loss from the lumbar spine. This was not attributable to our densitometric method that has a precision as good as or better than that of other methods of vertebral measurement and, using it, we were clearly able to detect premenopausal bone loss. Because we defined menopause in terms of both menstrual status and serum estrogen levels, we could control for the possibility that a gradual onset of estrogen deficiency associated with natural menopause resulted in a gradual onset in the postmenopausal acceleration of bone loss. We found that serum concentrations of estrone and estradiol did not decrease until after menopause, despite substantial vertebral bone loss in the premenopausal women. We cannot exclude the 
possibility, however, that small decreases in circulating sex steroids before menopause might have been detected had we assessed integrated serum concentrations over the entire menstrual cycle or assessed production rates.

We believe the most likely explanation is that the duration and extent of accelerated bone loss from the axial skeleton after menopause is less than has been generally believed and thus could not be detected by our study protocol. When Genant, Cann, and co-workers $(25,27)$ used computed tomography to measure BMD of the entire lumbar vertebra (which gives values comparable to those we obtain with dual-photon absorptiometry), they found the rate of integral bone loss was $6 \% / y r$ for the first year after oophorectomy and $2.5 \%$ for the second year. Only two of our subjects were followed transmenopausally and only four had baseline measurements made in the second year after menopause when vertebral bone loss may have already fallen from the high first-year rate to a rate only about twice that we observed in premenopausal women.

Thus, the rate and duration of accelerated bone loss following menopause may differ for the axial and for the appendicular skeleton. For the former, there may be large losses initially, which soon decline to approach the underlying premenopausal rate of bone loss after only a few years. For the latter, the accelerated phase of postmenopausal bone loss may have an initial rate that is less but a duration that is more prolonged. More experimental studies are needed to validate this hypothesis; in particular, longitudinal densitometric measurements of the axial and appendicular skeleton in women followed across natural menopause.

In conclusion, our longitudinal studies have demonstrated striking differences in the patterns of appendicular and axial bone loss over life in women. Only a small amount of cortical bone in the appendicular skeleton is lost before menopause, but large amounts are lost thereafter. By contrast, vertebral bone loss begins well before menopause and continues into old age with a trend toward midlife acceleration. Because our data show that almost half of the total bone loss occurs before serum estrogen levels decline, the menopause cannot be the only cause of vertebral osteoporosis. These findings suggest a need to identify factors responsible for premenopausal bone loss and, ultimately, to begin prophylactic intervention well before the menopause.

\section{Acknowledgments}

We are grateful to Dr. Stephen Kan for his assistance in collating and analyzing the data, to William $\mathrm{L}$. Dunn for collecting the bone densitometric data, and to Cheryl $\mathrm{K}$. Collins for typing the manuscript.

\section{References}

1. Riggs, B. L., and L. J. Melton III. 1983. Evidence for two distinct syndromes of involutional osteoporosis. Am. J. Med. 75:899-901.

2. Mazess, R. B. 1982. On aging bone loss. Clin. Orthop. Relat. Res. 165:239-252.

3. Riggs, B. L., H. W. Wahner, E. Seeman, K. P. Offord, W. L. Dunn, R. B. Mazess, K. A. Johnson, and L. J. Melton III. 1982. Changes in bone mineral density of the proximal femur and spine with aging. $J$. Clin. Invest. 70:716-723.

4. Mazess, R. B. 1983. The noninvasive measurement of skeletal mass. In Bone and Mineral Research, Annual 1. W. A. Peck, editor. Excerpta Medica/Amsterdam. 223-279.

5. Wahner, H. W., B. L. Riggs, and J. W. Beabout. 1977. Diagnosis of osteoporosis: usefulness of photon absorptiometry at the radius. $J$. Nucl. Med. 18:432-437.

6. Riggs, B. L., H. W. Wahner, W. L. Dunn, R. B. Mazess, K. P.
Offord, and L. J. Melton III. 1981. Differential changes in bone mineral density of the appendicular skeleton with aging: relationship to spinal osteoporosis. J. Clin. Invest. 67:328-335.

7. Judd, H. L., G. E. Judd, W. E. Lucas, and S. S. C. Yen. 1974. Endocrine function of the postmenopausal ovary: concentrations of androgens and estrogens in ovarian and peripheral vein blood. J. Clin. Endocrinol. Metab. 39:1020-1024.

8. Rosner, W. 1972. A simplified method for the quantitative determination of testosterone-estradiol-binding globulin activity in human plasma. J. Clin. Endocrinol. Metab. 34:983.

9. Statistical Analysis System User's Guide. 1979. J. Helwig and K. A. Council, editors. SAS Institute, Inc., Raleigh, NC.

10. Mazess, R. B., W. W. Peppler, R. W. Chesney, T. A. Lange, U. Lindgren, and E. Smith, Jr. 1984. Does bone measurement on the radius indicate skeletal status? Concise communication. J. Nucl. Med. 25:281288.

11. Smith, D. M., M. R. A. Khairi, J. Norton, and C. C. Johnston. 1976. Age and activity effects on rate of bone mineral loss. J. Clin. Invest. 58:716-721.

12. Hui, S. L., P. S. Wiske, J. A. Norton, and C. C. Johnston. 1982. A prospective study of change in bone mass with age in postmenopausal women. J. Chronic Dis. 35:715-725.

13. Krolner, B., and S. P. Nielsen. 1982. Bone mineral content of the lumbar spine in normal and osteoporotic women: cross-sectional and longitudinal studies. Clin. Sci. (Lond). 62:329-336.

14. Cann, C. E., H. K. Genant, F. O. Kolb, and B. Ettinger. 1985. Quantitative computed tomography for prediction of vertebral fracture risk. Metab. Bone Dis. Relat. Res. 6:1-7.

15. Meunier, P., P. Courpron, C. Edouard, J. Bernard, J. Bringuier, and G. Vignon. 1973. Physiological senile involution and pathological rarefaction of bone. Clin. Endocrinol. Metab. 2:239.

16. Merz, W. A., and R. K. Schenk. 1970. A quantitative histological study on bone formation in human cancellous bone. Acta Anat. 76:115.

17. Marcus, R., J. Kosek, A. Pfefferbaum, and A. Horning. 1983. Age-related loss of trabecular bone in premenopausal women: a biopsy study. Calcif. Tissue Int. 35:406-409.

18. Weaver, J. K, and J. Chalmers. 1966. Cancellous bone; its strength and changes with aging and an evaluation of some methods for measuring its mineral content. I. Age changes in cancellous bone. J. Bone Joint Surg. 48A:289.

19. Arnold, J. S. 1973. Amount of quality of trabecular bone in osteoporotic and vertebral fractures. Clin. Endocrinol. Metab. 2:221238.

20. Aloia, J. F., A. Vaswani, K. Ellis, K. Yuen, and S. H. Cohn. 1985. A model for involutional bone loss. J. Lab. Clin. Med. 106:630637.

21. Ruegsegger, P., M. A. Dambacher, M. S. Ruegsegger, J. A. Fischer, and $M$. Anliker. 1984. Bone loss in premenopausal and postmenopausal women. J. Bone Joint Surg. 66A:1015-1023.

22. Davis, M. E., N. M. Strandjord, and L. H. Lanzl. 1966. Estrogens and the aging process: The detection, prevention, and retardation of osteoporosis. J. Am. Med. Assoc. 196:219-224.

23. Recker, R. R., P. D. Saville, and R. P. Heaney. 1977. Effect of estrogens and calcium carbonate on bone loss in postmenopausal women. Ann. Intern. Med. 87:649-655.

24. Lindsay, R., D. M. Hart, C. Forrest, and C. Baird. 1980. Prevention of spinal osteoporosis in oophorectomised women. Lancet. ii: 1151-1154.

25. Genant, H. K., C. E. Cann, B. Ettinger, and G. S. Gordan. 1982. Quantitative computed tomography of vertebral spongiosa: a sensitive method for detecting early bone loss after oophorectomy. Ann. Intern. Med. 97:699-705.

26. Richelson, L. S., H. W. Wahner, L. J. Melton III, and B. L. Riggs. 1984. Relative contributions of aging and estrogen deficiency to postmenopausal bone loss. N. Engl. J. Med. 311:1273-1275.

27. Cann, C. E., H. K. Genant, B. Ettinger, and G. S. Gordan. 1980. Spinal mineral loss in oophorectomized women. J. Am. Med. Assoc. 244:2056-2059. 